Draft version MAy 6, 2016

Preprint typeset using $\mathrm{L}^{A} \mathrm{~T}_{\mathrm{E}} \mathrm{X}$ style emulateapj v. 01/23/15

\title{
LONG-TERM AND LARGE-SCALE HYDRODYNAMICAL SIMULATIONS OF MIGRATING PLANETS
}

\author{
Pablo Benítez-Llambay, Ximena S. Ramos, and Cristian Beaugé \\ Instituto de Astronomía Teórica y Experimental (IATE), Observatorio Astronómico, Universidad Nacional de Córdoba. Laprida 854, \\ X5000BGR, Córdoba, Argentina \\ FRÉDÉRIC S. MASSET \\ Instituto de Ciencias Físicas, Universidad Nacional Autónoma de México (UNAM), Apdo. Postal 48-3,62251-Cuernavaca, Morelos, \\ México \\ Draft version May 6, 2016
}

\begin{abstract}
We present a new method that allows long-term and large-scale hydrodynamical simulations of migrating planets over a grid-based Eulerian code. This technique, which consists in a remapping of the disk by tracking the planetary migration, enables runs of migrating planets over a time comparable to the age of protoplanetary disks. This method also has the potential to address efficiently problems related with migration of multi-planet systems in gaseous disks, and to improve current results of migration of massive planets by including global viscous evolution as well as detailed studies of the co-orbital region during migration.

We perform different tests using the public code FARGO3D to validate this method and compare its results with those obtained using a classical fixed grid.
\end{abstract}

Subject headings: planet-disk interactions - hydrodynamics - methods: numerical

\section{INTRODUCTION}

Giant planets form in gaseous environments, from which they acquire most of their mass. The interaction between both components, solids and gas, is extremely complex and one of the key aspects in any planetary formation theory. A planet immersed in a disk will accrete gas and experience changes in its mass, temperature and chemical composition. However, gravitational interactions are also important, as they exert a force onto the planet which dramatically alters its orbit, through an exchange of energy and angular momentum between the disk and the planet.

The gravitational force due to a planetary embryo excites spiral waves in the disk. These are launched at certain specific regions, known as Lindblad resonances, which combine to form a large scale asymmetric spiral wake co-rotating with the planet (Ward 1986, Ogilvie \& Lubow 2002, Rafikov 2002). Additionally, material trapped in the horse-shoe region also contributes to the exchange of angular momentum. This so-called corotation torque is typically of same order of magnitude as the torque produced by the spiral wake (Baruteau \& Masset 2013, and references therein). The net effect, considering both Lindblad and co-rotational torques, yields a time variation of the planet's semi-major axis usually referred to as type I migration.

The total gravitational torque between the gas disk and a low mass planet can be estimated analytically under certain simplifications, giving important insights about the physical mechanisms involved in migration (e.g. Goldreich \& Tremaine 1979, Tanaka et al. 2002, Baruteau \& Masset 2008a: Paardekooper \& Papaloizou 2009). How-

pbllambay@oac.unc.edu.ar xramos@oac.unc.edu.ar beauge凹ิoac.unc.edu.ar masset@icf.unam.mx ever, in the past two decades it has been increasingly clear that numerical experiments are indispensable in order to advance in migration theories.

Hydrodynamical simulations are computationally demanding and extremely costly since they must: (i) resolve the narrow region near the planet's orbit for a proper calculation of the co-rotation torque, (ii) maintain a good resolution far from the planet to resolve the spiral wake, and (iii) consider the entire azimuthal extension of the orbit to adequately describe the horseshoe motion.

Significant efforts have been made to gain in efficiency and realism, either by developing new algorithms to improve the orbital advection (Masset 2000) and/or by considering a self-consistent disk evolution during migration (Crida et al.2007). In particular, this second feature has been shown to be crucial in studies of the so-called type II migration, which occurs when the planet is massive enough to open a gap around its horseshoe region.

Recent exoplanetary detections, particularly by the Kepler mission, show the existence of many multiplanetary systems close to the star, some of which are located near (but outside) mean-motion resonances (Lissauer et al. 2011; Fabrycky et al. 2014). It is yet unclear whether these systems were formed in-situ (e.g. Hansen \& Murray 2013) or transported from beyond the ice line as a result of disk-planet interactions (e.g. Baruteau \& Papaloizou 2013).

Hydro-simulations of multiple planets face two challenges. On one hand, they must be able to follow the orbital decay from initial semi-major axes $a \sim 3 \mathrm{AU}$ down to final configurations with $a \sim 0.1 \mathrm{AU}$. In other words, simulations must be large-scale. On the other hand, even after the bodies reach the region close to the central star, their orbital evolution must be extended over a sufficiently long time scale to allow for the res- 
onant dynamics to act and guarantee that the outcome actually corresponds to a stationary solution. Thus, simulations must not only be large-scale but also long-term.

So far, hydro-codes have not been able to comply with these requirements. One of the main difficulties is caused by the Courant-Friedrich-Levy (CFL) condition, a stability constrain inherent to all explicit hydrodynamic solvers. In a cylindrical or spherical mesh, the CFL condition generally limits the time step through the size of the innermost cell. As a result, a gas disk that is modeled to include the regions close to the star will have a very small step size, even if the planets are initially located at large semi-major axis and if an orbital advection algorithm is used. Thus, radially extended and long-term numerical experiments have been practically impossible, and all simulations of planetary migration have been local, both with respect to space and time, with a few particular exceptions (e.g. Martin et al. 2007, Crida et al. 2009).

In this paper we present a new numerical method that overcomes these limitations. It basically consists of a remapping technique, which updates the mesh boundaries of the disk according the location of the migrating planets. The redefinition of the boundaries is automatic, leading to high-resolution simulations that are independent of the orbital evolution of the system. Moreover, since the mesh is not defined in regions that are not required, the code gains in efficiency and precision, allowing simulations virtually infinite in time and space.

Our paper is organized as follows: in section 2 we present the standard disk model used for this work, in section 3 we present the remapping method, in section 4 we present different tests used to validate our implementation. In section 5 we discuss the benefits of our method compared with the current techniques. In section 6 we present two simulations including 2 and 3 planets showing the capabilities of the method. In section 7 we present a final discussion and the conclusions of this work.

\section{DISK MODEL}

We describe the problem using the Navier-Stokes equations in a non-rotating inertial frame. For simplicity we solve these equations integrated over $z$, assuming a two dimensional non self-gravity gas disk orbiting a central star of mass $M_{*}$. Continuity equation reads:

$$
\frac{\partial \Sigma}{\partial t}+\nabla \cdot(\Sigma \vec{v})=0
$$

where $\Sigma$ is the surface density, and $\vec{v}$ is the velocity field of the fluid. The momentum equations are:

$$
\rho\left(\frac{\partial \vec{v}}{\partial t}+\vec{v} \cdot \nabla \vec{v}\right)=-\nabla P-\rho \nabla \vec{\phi}+\nabla \cdot \vec{\Pi}
$$

where $P$ is the pressure and $\phi$ is the gravitational potential, which includes the contribution of the star and planets. $\vec{\Pi}$ is the stress tensor, given by:

$$
\vec{\Pi}=\rho \nu\left[\nabla \vec{v}+(\nabla \vec{v})^{T}-\frac{2}{3}(\nabla \cdot \vec{v}) \vec{I}\right],
$$

with $\nu$ the kinematic viscosity and $\vec{I}$ the identity tensor.

We use a locally isothermal equation of state, in which the gas pressure is related to the surface density by $P=$ $c_{s}^{2}(r) \Sigma$, with $c_{s}$ the sound speed of the gas. $c_{s}$ is related with the aspect ratio $h$ of the disk by $c_{s}=r \Omega_{k} h(r)$, which is given in term of the vertical disk scale height $H$ by $h(r)=H(r) / r$. The Keplerian angular velocity is $\Omega_{k}$, given by $\Omega_{k}=\sqrt{G M_{*} / r^{3}}$, where $G$ is the gravitational constant and $r$ denotes the distance to the central star.

In our model, we adopt power laws for $\Sigma$ and the aspect ratio $h$ of the disk, with indices $\alpha$ and $f$ respectively:

$$
\Sigma \propto r^{\alpha} \text { and } h \propto r^{f} .
$$

This implies a power law for the temperature $T$, with index $\beta=2 f-1$. The planet of mass $m_{p}$ orbits the central star and feels the gravitational potential of the disk. It has a softened potential of the form:

$$
\phi_{p}=-\frac{G M_{*}}{\sqrt{\left|r-r_{p}\right|^{2}+\epsilon^{2}}},
$$

where $\epsilon$ is the softening length. We use a softening length parametrized by the pressure scale height in the form $\epsilon=0.6 H$. We neglect the indirect terms since they are not relevant to our concerns.

Damping boundary conditions are applied following de Val-Borro et al. (2006). These are required to match the large-scale and long-term behavior of the disk, given either by power laws or by an auxiliary one-dimensional calculation. We use the hydrodynamical code FARGO3D (Benítez-Llambay \& Masset 2016) over a polar mesh evenly spaced in radius and azimuth.

\section{METHOD AND NUMERICAL IMPLEMENTATION}

The main idea behind our method is to take a previous state as a spatially re-sampled initial condition to advance the system into a new state. There are two possible ways to do this. One way is to integrate the system to a specific state and then re-sample the physical quantities and restart the simulation. A second possibility is to use a continuous recipe, where for each time step the mesh is re-sampled. We choose the second option since it allows for smooth changes of all quantities.

Since we are interested in following planets while they migrate, the re-sampling must be performed exclusively radially. The algorithm we developed has three basic steps, consisting of a suitable recipe to calculate the new radial borders of the mesh, a method to re-fill the mesh with appropriate values for the hydrodynamic quantities and, finally, an update of the boundary conditions and damping zones.

To find the radial borders for the new mesh we use the planets' location. The gas ring furthest from the planet that is able to exert a torque is located at the inner (outer) 2:1 (1:2) mean motion resonance (MMR) with the planet (Goldreich \& Tremaine 1979), which are the furthest Lindblad resonances with respect to the planet. Thus, the new border positions should be calculated as a function of MMRs or period ratios with the planet closest to them. In this way we ensure to incorporate all the ingredients of type I migration during the entire simulation.

Once we have computed the new border positions, we radially split the mesh using the same number of cells than in the previous step. This choice is motivated by the simplicity of implementation. 


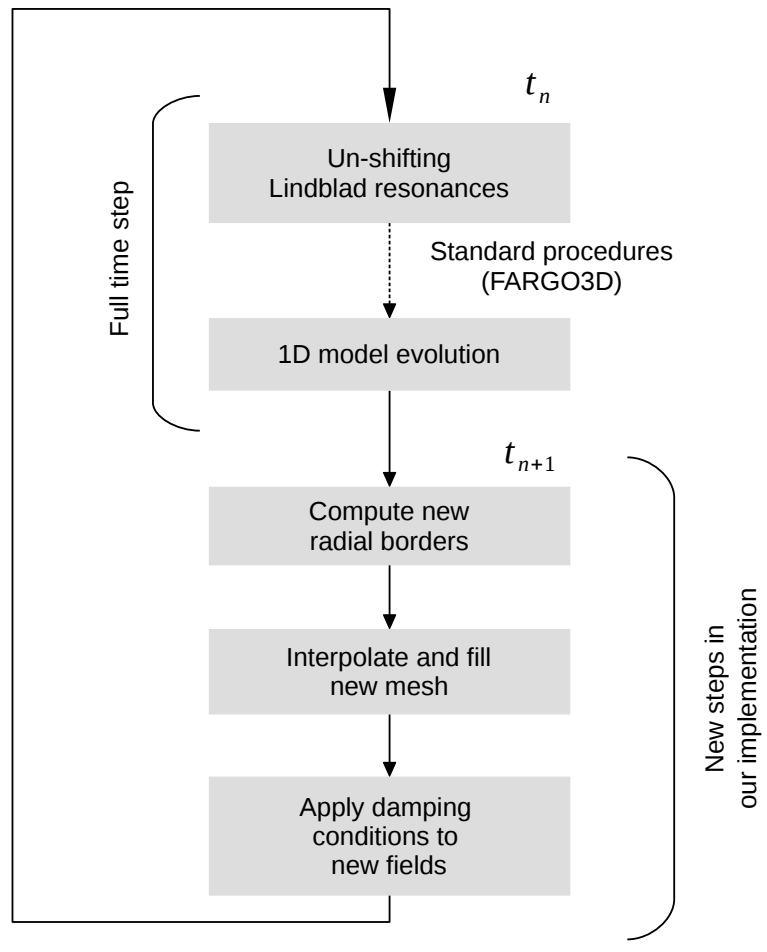

FIG. 1. - Flow chart showing how our implementation is coupled to the standard flow of FARGO3D. For simplicity we show only the part corresponding to a full update (see figure 2 in BenítezLlambay \& Masset (2016) for details). Inside the main loop, we tollow the method described in $\$ 3.4$ for un-shifting the Lindblad Resonances and we use the time step already calculated by the CFL condition to advance the $1 \mathrm{D}$ model for the surface density under the effect of the viscous evolution of the disk (see $\sqrt{3.3}$. After that, a complete time step is performed for all fields. Then, we recompute the new location of the borders of the mesh. Finally, before advancing another time step, we update the damping zones. All fields are damped toward the updated reference values, given by the prescriptions detailed in 3.2

Finally, we perform a linear interpolation, using the old fields, to calculate the values at the position of the new cells. If the new cells fall outside the old domain, we extrapolate the fields using analytical prescriptions given either by initial conditions or by an auxiliary viscous evolution model (see 3.3 . .

In Fig. 1 we present a simple flow chart showing how the remapping algorithm is implemented.

\subsection{Defining the new borders}

To define the borders for the mesh we have to consider two different zones: (i) the active mesh (where there is no damping), and (ii) the damping zones.

The active mesh is defined in terms of period ratios with respect to the planets located in the mesh:

$$
r_{\mathrm{d}}^{ \pm}=r_{p}^{ \pm} \mathcal{R}_{\mathrm{d}}^{ \pm 2 / 3}
$$

where $r_{p}^{ \pm}$is the semi-major axis of the closest planet to the \pm border and $\mathcal{R}_{\mathrm{d}}$ is a positive real number greater than one, which represents the period ratio between the planet and the gas at the border of the active mesh (without considering the damping zones).

Once we have determined the location of the active mesh, we proceed to calculate the total extension of the mesh. Since the characteristic time for damping is nor-

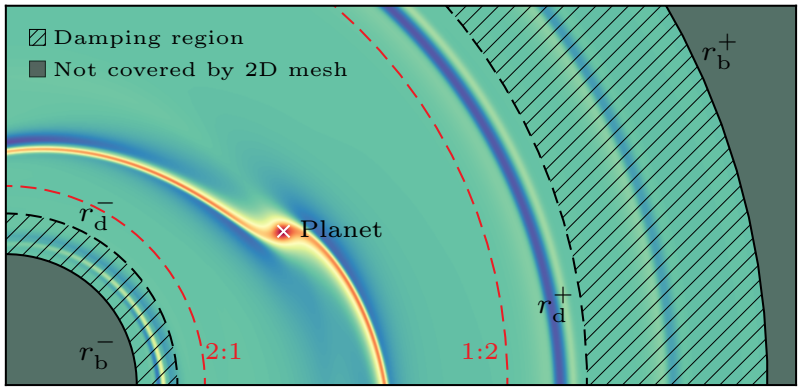

FIG. 2.- A wedge of a typical perturbation of surface density. The gray zone marks the region of the disk where the $2 \mathrm{D}$ mesh is undefined. Black continuous lines represent the border of the active mesh. The hatched zones show where damping boundary conditions are applied to the fields. They are bounded by black dashed lines. The mesh portion that extends from the inner to the outer black dashed lines is referred as the active mesh. In the case shown here, we set up the dashed lines using Eq.66 with $\mathcal{R}_{\mathrm{d}}=$ $5 / 2$. For damping zones, the distance from the first ring to the corresponding border of the mesh is computed using Eq. 8 with $\mathcal{R}_{\mathrm{b}}=3 / 2$. In red dashed lines we show the location of the $2: 1 / 1: 2$ MMRs with the planet, which are important locations because only between them the gas exert an effective torque onto the planet, marked by a white cross.

mally chosen proportional to the local orbital period of the damping cells (de Val-Borro et al.2006), it is natural to define its width so as to span a fixed extent in orbital periods, in the same way as was done to define $\mathcal{R}_{\mathrm{d}}$ :

$$
r_{\mathrm{b}}^{ \pm}=r_{\mathrm{d}}^{ \pm} \mathcal{R}_{\mathrm{b}}^{ \pm 2 / 3}
$$

where $\mathcal{R}_{\mathrm{b}}$ is a positive real number greater than one, which represents the period ratio between the first damping ring and the border of the mesh.

Using Eqs. (6) and (7) we obtain the location of the borders that keeps a constant size in periods with respect to the planet, given by:

$$
r_{\mathrm{b}}^{ \pm}=r_{p}^{ \pm}\left(\mathcal{R}_{\mathrm{d}} \mathcal{R}_{\mathrm{b}}\right)^{ \pm 2 / 3} .
$$

In Fig. 2, we show a sketch of a wedge for a typical simulation. The damping regions are represented by the hatched zones and their limits indicated by dashed black lines, whose location is determined by Eq. (6). The limits of the 2D mesh (black continuous lines) are given by Eq. (8). Red dashed lines show the location of the 2:1/1:2 MMR between gas and the planet. In this particular case, we have chosen the values $\mathcal{R}_{\mathrm{b}}=3 / 2$ and $\mathcal{R}_{\mathrm{d}}=5 / 2$.

Fig. 3 shows the evolution of the semi-major axis of a low mass planet for a typical simulation. The mesh is modified continuously at runtime to keep the borders at a fixed period ratio with the planet, so as to track it. The mesh is shown by the blue region, and its extension can be calculated by using Eq. (8).

\subsection{Filling the mesh}

Once we have computed the new borders, we radially split the mesh using the same number $n_{r}$ of radial cells as in the previous state. In this work we use an even spacing between cells, but any other spacing is possible, such as a logarithmic spacing. A further discussion on this choice is given in $\$ 4.5$.

For each new cell, we calculate its neighbors on the old mesh and use them to estimate the new values using 


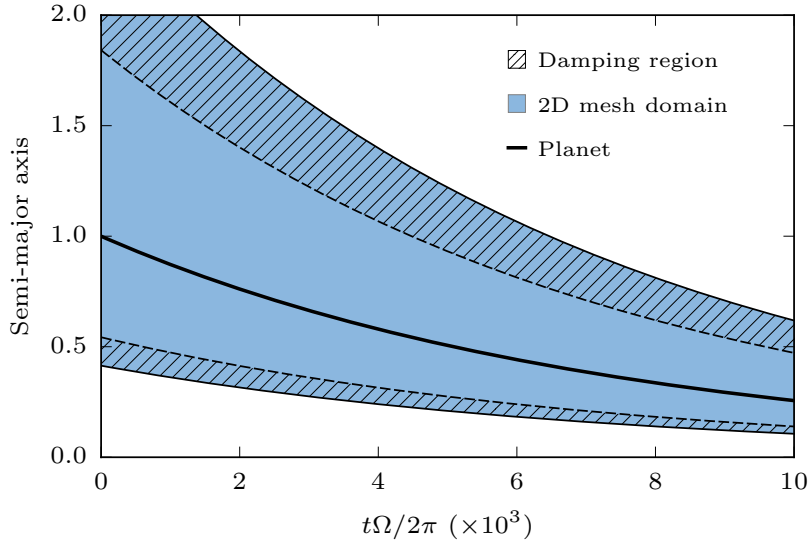

FIG. 3.- Semi-major axis evolution of a planet (thick black line) using the remapping method. The blue region correspond to the extension of the 2D mesh. Damping regions are hatched.

\section{linear interpolation.}

If two neighbors are not available, or if the updated cell falls onto the damping rings, we use analytical prescriptions to compute the corresponding values. These prescriptions may be given by the initial conditions for the sound speed (or temperature) and the azimuthal velocity. Similarly, the initial conditions are used to prescribe the surface density and radial velocity if the viscous evolution of the disk is not taken into account; otherwise, as we will see in $\$ 3.3$ we use Eq. (12) to update the surface density and Eq. (13) to get the radial velocity.

Now we will discuss the basic algorithm for the case in where the mesh is linearly spaced.

We start from a quantity $q$ at location $r_{i}$ at time $t_{n}$, and denoted by $q_{i}^{n}$. After a time step, the new updated value $q_{i}^{n+1}$ is obtained by solving the hydrodynamics equations using finite difference upwind, dimensionally split methods, combined with the FARGO algorithm (Masset 2000) for orbital advection and a fifth order Runge-Kutta integrator for advancing the planets (Benítez-Llambay \& Masset 2016).

The border locations are updated, from $\left(r_{b}^{-}\right)^{n},\left(r_{b}^{+}\right)^{n}$ to $\left(r_{b}^{-}\right)^{n+1},\left(r_{b}^{+}\right)^{n+1}$ following the recipe described by Eq. (8). After that, we need to compute the new radial ocations $r_{j}$ for each cell. In the evenly (or linearly) spaced case, this can be done using the simple relation $r_{j}=\left(r_{b}^{-}\right)^{n+1}+j \Delta, j=0 \ldots n_{r}-1$, and $\Delta=\left[\left(r_{b}^{+}\right)^{n+1}-\left(r_{b}^{-}\right)^{n+1}\right] / n_{r}$. For the sake of definiteness, we will assume that $q$ is a face-centered (i.e. staggered) quantity, such as $v_{r}$, the radial velocity. Its value after remapping is given by:

$$
q_{j}^{n+1}=q_{k_{j}}^{n+1}+\left.\frac{q_{k_{j}+1}-q_{k_{j}}}{r_{k_{j}+1}-r_{k_{j}}}\right|^{n+1}\left(r_{j}-r_{k}\right),
$$

with $k_{j}$ a function of the old spacing. In the linearly spaced case, the index $k_{j}$ is given by:

$$
k_{j}=\operatorname{int}\left[\frac{r_{j}-\left(r_{b}^{-}\right)^{n}}{\left(r_{b}^{+}\right)^{n}-\left(r_{b}^{-}\right)^{n}} n_{r}\right],
$$

where $\operatorname{int}(x)$ represents the largest integer inferior to $x$. The extension to the case of cell-centered variables is

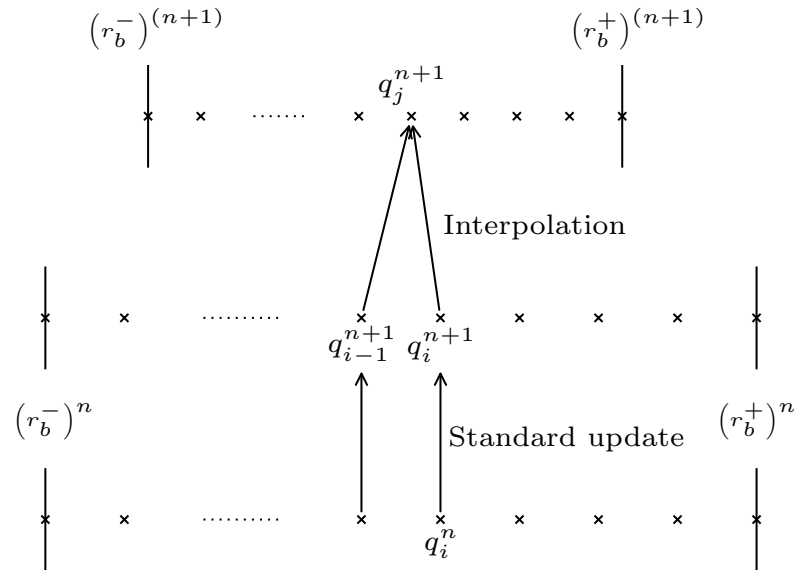

FIG. 4.- Diagram showing how a quantity $q_{j}^{n+1}$ in the remapped mesh is obtained from old values. In the bottom part we start at time $t_{n}$ and update the quantity $q_{i}^{n}$ to a new value $q_{i}^{n+1}$ (middle step) by evolving the hydrodynamics equations. Finally, at the top part we compute the new borders of the mesh and we fill it at location $r_{j}$ by interpolating the recently updated field.

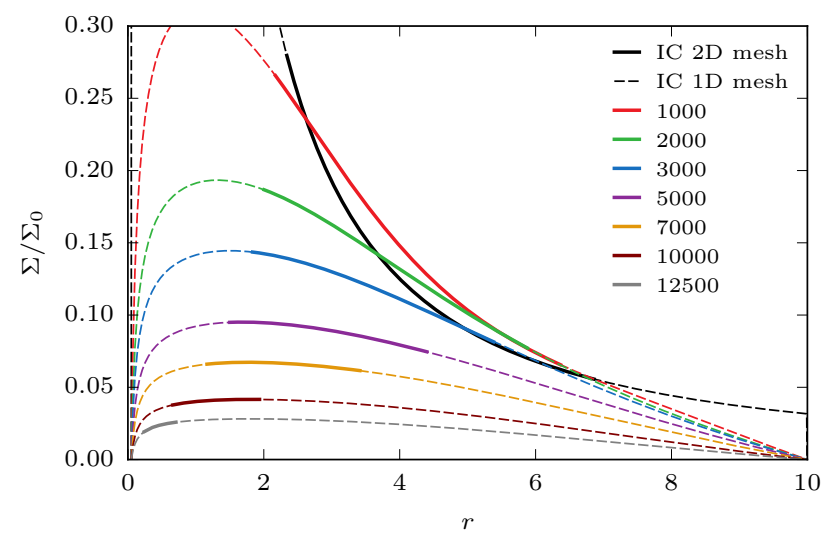

FIG. 5.- Viscous evolution of a surface density profile initially given by a power law $\Sigma \propto r^{-1.5}$ with no planet. The number of cells used for the 1D viscous model (Eq. 12) is 2048 while the 2D mesh has 256 radial zones. The viscosity $\nu$ is constant over the entire domain and a zero surface density boundary condition is applied at $r=0.05$ and $r=10$. The $2 \mathrm{D}$ mesh has an imposed, constant migration rate. Labels are given in orbital periods at $r=1$.

straightforward. In Fig. 4 we depict this algorithm, and we put in context all important quantities.

\subsection{Viscous evolution of the disk}

Since the remapping method allows to perform longterm simulations over a wide spatial range, special considerations regarding the global viscous evolution of the disk should be taken into account. Describing a disk with a power law for long-term simulations may not be an appropriate choice. From the third term in the right hand side of Eq. (2), the entire disk is expected to drift over a time scale $\tau_{\nu} \propto r^{2} / \nu$ (Pringle 1981). On the other hand, the characteristic time scale for migration $\left(\tau_{a}=r_{p} / \dot{r}_{p}\right)$ in the type I regime scales as $\tau_{a} \propto M_{*}^{2}(h / r)^{2}\left(m_{p} \Sigma\right)^{-1} \Omega^{-1}$ Ward (1986). 
Thus, the ratio between both time scales is given by:

$$
\frac{\tau_{a}}{\tau_{\nu}} \propto \frac{M_{*}^{2} h^{2} \nu}{m_{p} \Omega \Sigma r^{4}} .
$$

At $r=R_{0}=5.2 \mathrm{AU}$, typical parameters are $h=0.05$, $\nu=10^{-5} R_{0}^{2} \Omega_{0}$ and $\Sigma \sim 6 \cdot 10^{-4} M_{\odot} R_{0}^{-2}$, and assuming $M_{*}=1 M_{\odot}, m_{p}=10^{-5} M_{\odot}$ it gives a ratio equal to $\sim 4$. This value depends on $r$ as $r^{-(2.5+\alpha)}$ (assuming $f=0$ ), where $\alpha$ is defined by Eq. (4), and in practice is a negative number greater than -1.5 . Thus, the migration rate becomes negligible compared to the viscous time as the planet migrates to the star. This simple analysis shows that in order to perform a consistent simulation of migrating planets in type I regime over a long scale (in space and time), a self-consistent viscous evolution of the disk should be considered. We address this issue by solving a simplified one-dimensional model for the angular momentum conservation of the disk under the effects of viscosity and neglecting gas pressure and planet torques (Pringle 1981):

$$
\frac{\partial \Sigma}{\partial t}=\frac{3}{r} \frac{\partial}{\partial r}\left[\sqrt{r} \frac{\partial}{\partial r}(\nu \Sigma \sqrt{r})\right]
$$

We evolve Eq. (12) explicitly over a sufficiently large one-dimensional radially extended mesh at runtime during the full two-dimensional simulation. We use the advanced surface density to update the boundaries at the damping regions. Once the surface density is calculated, the radial velocity can be inferred by the closed relation:

$$
v_{r}=-\frac{3}{\Sigma \sqrt{r}} \frac{\partial}{\partial r}(\nu \Sigma \sqrt{r}) .
$$

This implementation of the viscous evolution for the disk by using a one-dimensional mesh can be compared to the implementation of Crida et al. (2007). In our case we treat reflections by using the well known damping zones (de Val-Borro et al. 2006) and we do not address the angular momentum conservation issue because we assume that planets do not strongly alter the surface density far away from its current location. This approach is not as sophisticated as that of Crida et al. (2007), but we will see in next sections that we can achieve very clean and accurate results. One important difference with their implementation is that the $2 \mathrm{D}$ mesh was fixed, so that planets could get close to the boundaries and some care had to be taken with the wake's momentum flux into the one-dimensional grid. Here our prescription of Eq. (8) allows planets to stay clear of the boundaries, and our damping boundary prescription is sufficient to ensure a smooth transition between the 2D and 1D meshes.

Fig. 5 shows an example of how the 2D migrating mesh (using the remapping method) can be altered by considering long-term simulations. In this figure can be compared each of the continuous lines with the expected solution given by the corresponding dashed line. This figure corresponds to the test shown in $\$ 4.3$.

\subsection{Un-shifting Lindblad resonances}

Baruteau \& Masset (2008b) show that a special treatment for computing the torque is needed when selfgravity is not included in simulations of migrating planets. They show that, due to a spurious shift of Lindblad

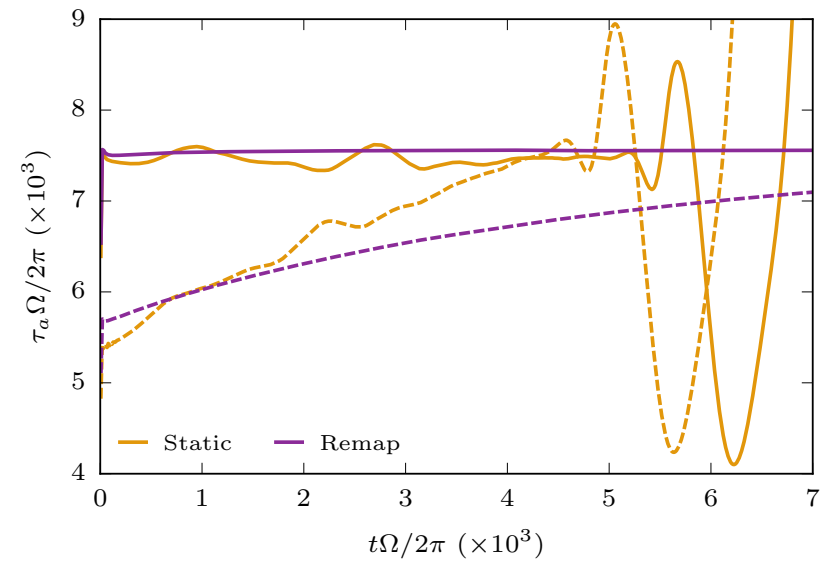

FIG. 6.- Characteristic migration time for the static (orange) and remapping (purple) cases. Continuous and dashed lines correspond to results with and without correcting for the spurious shift of Lindblad resonances respectively. In agreement with Baruteau \& Masset (2008b), the migration time scale is shorter when the planet and the disk do not orbit the same potential.

resonances, a migrating planet in a non-self gravity disk migrates faster than theoretical predictions and in fully self-gravity simulations.

This spurious shift occurs because planet and disk orbit a different effective potential, since the planet feels the disk gravity but the disk does not feel itself.

The spurious resonance shifting can be easily addressed by subtracting the azimuthal mean density prior to the force calculation. This method does not impact on the net torque, but changes the orbital frequency of the planet, fixing the shift and ensuring an accurate migration rate.

To compute the gravitational force first, we subtract the mean surface density at time $t_{n}$ :

$$
\delta \Sigma(r, \varphi)=\Sigma(r, \varphi)-<\Sigma>_{\varphi}
$$

where $\varphi$ is the azimuthal coordinate and $\langle\Sigma\rangle_{\varphi}$ is defined as:

$$
<\Sigma>_{\varphi}=\frac{1}{n_{\varphi}} \sum_{i=0}^{n_{\varphi}-1} \Sigma\left(r, \varphi_{i}\right),
$$

and we calculate the gravitational force using $\delta \Sigma$.

To our concerns, this shift is extremely important to obtain convergent migration rates, as can be seen in Fig. 6, corresponding to the test of $\S 4.4$. By considering different radial extensions for the mesh (at a fixed resolution), we are artificially including a different amount of mass in the inner regions of the disk. This changes the planet's orbital frequency with respect to the disk and directly impacts the net torque exerted onto the planet. This effect is clearly shown by the purple dashed line of Fig. 6, where the asymptotic value, corresponding the a narrow and low mass mesh, tends to its respective fixed case (purple continuous line).

\section{NUMERICAL TESTS}

In this section we show a set of tests to validate the remapping method. Runs presented here include all the features already described: (i) the remapping method, (ii) the global viscous evolution and (iii) the shift to the Lindblad resonances. For comparison purposes we do not 
include a certain feature in some tests, like the shifting for the Lindblad resonances and the remapping method for fixed-mesh simulations.

We used the public code FARGO3D $\mathrm{D}^{1}$ adapted to include our method using the disk model presented in \$2. Our mass unit is $M_{*}=1$, our length unit is $R_{0}$, the planet's initial orbital radius, and our time unit is $t=\Omega_{k}^{-1}$ (in this unit system we have therefore $G=1$ ). We consider a planet of mass $m_{p}=2 \times 10^{-5} M_{*}$ and a gaseous disk of parameters $\Sigma\left(R_{0}\right)=10^{-3}, \alpha=-0.5$, $h\left(R_{0}\right)=0.05, f=0$. We use the prescription of Shakura \& Sunyaev (1973) for viscosity, defined by $\nu=\alpha_{\nu} H^{2} \Omega$, with a constant value $\alpha_{\nu}=4 \times 10^{-3}$. The azimuthal domain is $[0,2 \pi]$. The radial domain will be described for each particular case. In the tests without remapping, the damping regions span a period extension equal to $3 / 2$ from each active border, using a prescription similar to that employed for the remapping case (Eq. (7)).

\subsection{Results without the remapping technique}

In this section we measure the migration rate of a single planet, considering a static (not remapped) disk. Both the borders and damping regions are assumed fixed. An important parameter involved in this test is the characteristic damping time. By numerical experiments we found that a good value to minimize reflections considering the adopted extension for the damping zone is $0.3 \Omega^{-1}$. The limits of the mesh were chosen as $R_{\min }=0.37$ and $R_{\max }=2.08$, and we consider three different resolutions: $n_{r} \times n_{\Phi}=462 \times 512,924 \times 1024$ and $1386 \times 1536$.

In Fig. 7 the evolution of the characteristic migration time for the three different cases is shown. A converged result is fairly achieved by the middle resolution case, as is shown by the blue and red curves.

We observe very small oscillations in the migration rate for the three cases, which don't depend strongly on resolution and can be explained as a reflection of the wake by the borders of the mesh. We have checked this assumption by performing simulations with different radial extension but at the same resolution. We have observed a shift of these oscillations when the radial extent changes. As we will see in the next sections, these oscillations are not present when we use the remapping method for an isolated planet and a non-flared disk (e.g. purple lines of Fig. 6). This does not mean that the remapping method allows to improve the value of the migration rate with respect to the standard case for a comparable resolution. It only means that the reflected wake maintains a fixed position with respect to the planet for the entire simulation. This assertion can be easily proved using the expression for the azimuthal position of the wake provided by Ogilvie \& Lubow (2002). According to these authors, the azimuthal angle of the wake scales linearly with the distance of the planet and $h$. Since our method sets the edges at a distance proportional to the planetary orbital radius, this angle remains constant.

Finally, from the amplitude of these oscillations, we can get an idea of the precision for the measured migration rates (not better than $3 \%$ in this case, which is extremely good). In principle, the precision achieved de-

\footnotetext{
1 http://fargo.in2p3.fr/
}

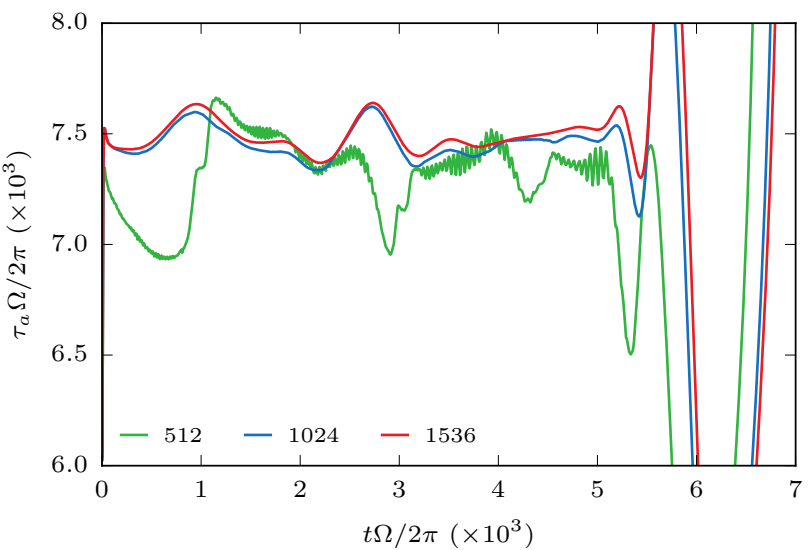

FIG. 7.- Characteristic migration time $\tau_{a}$ for three different simulations considering a static mesh (without the remapping method) but considering different resolutions: the green continuous line correspond to the lower resolution case, the blue continuous line doubles it and the red one increases it by a factor 3 . The three curves shown a similar overall behavior. Higher oscillations are present in the lower resolution case. At time $\sim 5.5 \times 10^{3}$ orbits curves diverge because the planet reach the inner limit of the mesh, and migration halts. As is described in the main text, oscillations can be attributable to reflections of the wake on both boundaries.

pends on how well reflection can be avoided, so we could modify the damping prescription to further decrease reflection and thus improve the measures.

At time $\sim 5.5 \times 10^{3}$ orbits all curves reach the inner boundary of the mesh, migration halts and migration rates diverge.

\subsection{Convergence tests with the remapping algorithm}

The remapping algorithm needs two different radial extents to be defined: (i) the active mesh and (ii) the damping zones, controlled by the dimensionless parameters $\mathcal{R}_{\mathrm{d}, \mathrm{b}}$. In this section we perform two tests to verify how well migration rates are measured by varying both of them. In all cases the characteristic time for damping was chosen as $0.3 \Omega_{k}^{-1}$.

The first test consists in simulating an isolated planet in a disk with the same parameters already described, but varying both the effective resolution and $\mathcal{R}_{\mathrm{d}}$ on a grid. We used three different resolutions, referred to by the number of azimuthal zones: 512,1024,1536, corresponding to the same resolutions used in the simulations performed without the remapping algorithm. We varied $\mathcal{R}_{\mathrm{d}}$ linearly from 1.5 to 3 by steps of 0.1 . We have run each test for at least $10^{3}$ orbits, and the values reported here are an average of the characteristic migration time over the last hundred of orbits (which is constant for these parameters).

The left panel of Fig. 8 shows the results of this test. The lower resolution case (in red) shows a systematic increase of the migration time, evidencing that this resolution is not sufficient to resolve the physical processes involved. The intermediate and high resolution cases (green and blue respectively) display a nearly converged rate. In this test we can see that the oscillations observed in the static case are still present when we measure the migration rate by considering different radial extents of the mesh. This feature is an evidence of reflections of the wake on the boundaries, producing a variable migration rate. The amplitude of these oscillations gives an 

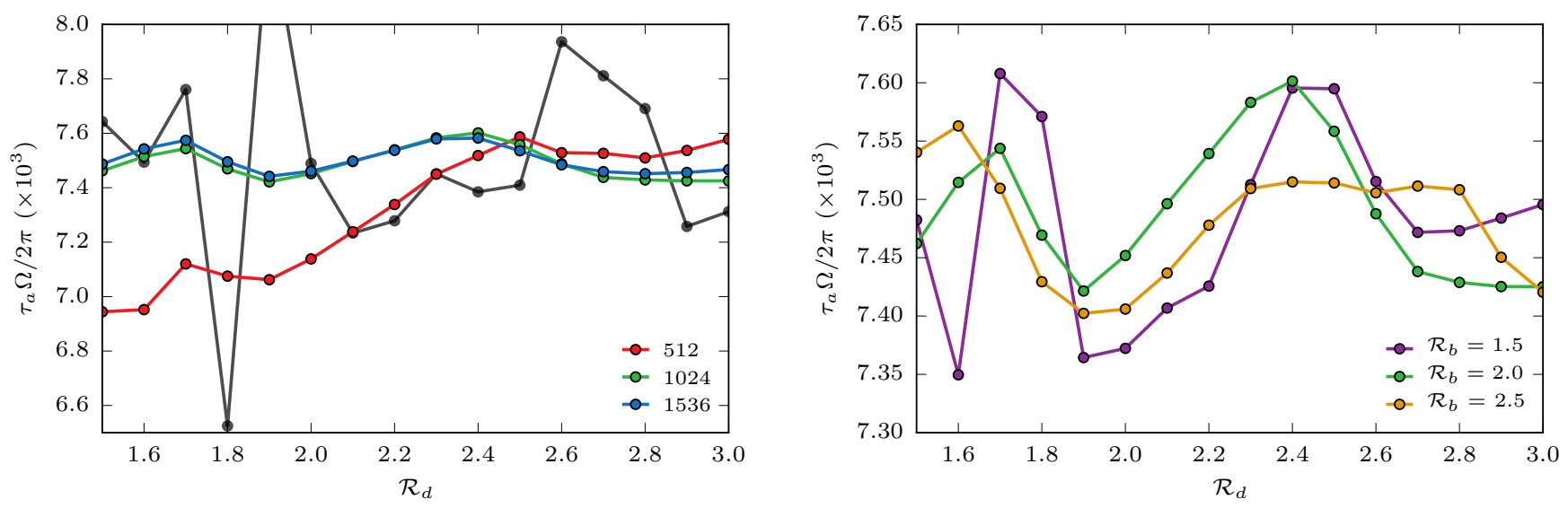

FIG. 8.- Characteristic migration time as a function of $\mathcal{R}_{\mathrm{d}}$ for the remapping case. The left panel shows a set of simulations with three different resolutions (referred to as 512,1024 and 1536) and same value $\mathcal{R}_{\mathrm{d}}=2$. The black curve shows a set of intermediate resolution with a stronger damping. The right panel shows a set of simulations with three different values for $\mathcal{R}_{\mathrm{b}}(1.5,2.0$ and 2.5$)$ for the intermediate resolution set. It is noticeable how the amplitude of the curves decreases for larger $\mathcal{R}_{\mathrm{b}}$.

estimate of the precision achieved (not better than $3 \%$ in this particular case). For all runs presented in this panel we have used $R_{b}=1.5$. Surprisingly, we do not observe an important variation of migration rate by considering $\mathcal{R}_{\mathrm{d}}<2.0$, probably due the smooth and large damping regions used. This parameter should nevertheless be greater than 2 to capture all Lindblad resonances. To test this idea we have run a set of similar simulations but defining a very strong damping condition over a narrow region, showed by the black curve. In this case we can see fast and strong variations in the migration rate for $\mathcal{R}_{\mathrm{d}}<2.0$. For $\mathcal{R}_{\mathrm{d}}>2.0$ we still observe oscillations with lower amplitudes, attributable to the reflection of the wake as we show below.

The second test was designed to prove that oscillations observed in the migration rate for different values of $\mathcal{R}_{\mathrm{d}}$ are consistent with reflections of the wake. To prove this statement we have varied the value of the parameter $\mathcal{R}_{\mathrm{b}}$, taking 1.5, 2.0 and 2.5 for the intermediate resolution run (green curve in left panel of Fig. 8). The results are presented in the right panel of Fig. 8. It is noticeable how all curves return similar values for the mean migration rate, but the extreme values are shifted. Another interesting feature is that the amplitude of oscillations decreases when $\mathcal{R}_{\mathrm{b}}$ increases. This is natural if we assume that these oscillations are due to reflections, since larger damping regions naturally better damp perturbations.

It is important to note that these tests have been done for a planet on a circular orbit. In the case one considers planets with a finite eccentricity, the size of the active mesh should be validated by a similar convergence test, since in such cases higher order Lindblad resonances (such as the 3:1 MMR), located even further from the planet's orbit, may play a non-negligible role in the planetary migration. Also, it could be important in some specific cases to take into account the physical size for the resonances, which have an effective width that should not be neglected. This size depends on the azimuthal wave number and reaches a maximum for low values (Artymowicz 1993).

\subsection{Viscous evolution test}

We carried out a viscous evolution test for an axisymmetric disk with no planet. We initialize a non-flared $(f=0)$ locally isothermal and power law surface density disk. We have used a uniform viscosity $\nu=10^{-4} R_{0}^{2} \Omega_{0}$.

The $1 \mathrm{D}$ viscous model, given by Eq. (12), is evolved using 2048 uniform radial zones between $r=0.05$ and $r=10$, with null surface density boundary conditions. The $2 \mathrm{D}$ mesh has 256 radial zones and the full radial extent spans over 3 periods in space $\left(\mathcal{R}_{\mathrm{d}, \mathrm{b}}=1.5\right)$. We radially move the mesh at a constant speed to test how the viscous method couples with the remapping one.

Fig. 5 shows the surface density evolution at different times. In continuous lines 2D surface density is shown. Dashed lines correspond to the solution of Eq. (12), used to update the boundaries and damping zones, for different times. In black, initial conditions are shown, while the evolution of the surface density, under the effects of viscosity, is presented in colored lines.

We have also checked that we are able to obtain a steady solution by considering an $\alpha_{\nu}$ disk model, with $\alpha=-0.5$ and $f=0$, configuration used in all the tests considering planets.

Results obtained are remarkably clean, allowing to perform large-scale migration of planets considering the global viscous disk evolution, and even open the possibility for long-term simulations of type II migration.

\subsection{Fixing orbital frequencies}

An important characteristic that we have included in our method is the correction for the Lindblad resonances spurious shifting. It is a crucial component for having converged simulations by using the remapping method, as was already described in 3.4 .

We have checked our implementation by using two different simulations set, one without the remapping method and the other one by considering all the ingredients. The parameters used are the same as those used in the fixed cases (see 4.1), but in the remapping case we consider $\mathcal{R}_{\mathrm{d}}=2.0$ and $\mathcal{R}_{\mathrm{b}}=1.5$. Both cases have same initial resolution.

We have computed the gravitational force including the mean background density and subtracting it, following Eq. (14. 
Fig. 6 shows the evolution for the characteristic migration time for these four simulations. In orange lines we show the simulations that do not include the remapping method while purple lines show the simulations that include it. Dashed lines correspond to the non-corrected cases and continuous lines correspond to the fixed ones.

As we evidenced in section 3.4 and in agreement with Baruteau \& Masset (2008b), the shifted cases (noncorrected) have a shorter migration time scale.

It is interesting to note that the non corrected case using the remapping method, has a non zero slope. It is possible to prove by linear calculations (Tanaka et al. 2002 , for instance), that this slope should be in fact flat for this parameter set. Thus, it clearly shows that migration rate achieved without the correction is not right. The same occur for the static case.

Another important feature of Fig. 6 is that both, the static and the remapping cases tend toward the corrected one at larger time. This is a consequence of having a narrow and virtually massless disk in the inner regions.

Finally we remark that in the remapping case we do not observe oscillations for the migration rate (for a one planet case). This feature can be explained as a constant shift between the reflected wake and the planet, as was already discussed in $\S 4.1$.

\subsection{Resolving the disk pressure scale}

A difficulty with the remapping method in the form presented here is the requirement of maintaining an overall sufficient resolution for a given disk model.

The pressure scale length of the disk should be well resolved at least near the planet, which also implies to resolve the closest Lindblad resonances, accumulating at $r \simeq \pm H$ (Artymowicz 1993).

In tests presented in this paper we have only considered a non-flared disk, implying $H \propto r$. In an evenly spaced mesh, a uniform sampling remapping method has a linearly increasing resolution with $r_{p}$, and the number of cells per unit of $H$ will be the same for the entire simulation, thus ensuring converged results for migration rates. However, when we consider a flared disk, the number of cells per unit of $H$ is $\propto r^{-f}$, which is a decreasing function for flared disks $(f>0)$. Thus, a growing number of cells per unit of $H$ is needed when the planet migrates to the star.

This highlights the need for non-uniform grids to simulate migrating planets in a flared disk, which ideally should be radially spaced as $\propto r^{f+1}$.

However, in real cases disks can not be flared over large radial range, limiting the maximum flare allowed. Thus, in practice a sufficiently resolved logarithmic migrating mesh coupled with a realistic disk model, like a nonglobal power law for density and or temperature, should be good enough to capture all important details of planetary migration for large-scale migration.

\section{IMPROVEMENTS AND CONSERVATION PROPERTIES}

If quantities are power laws of radius, such as the surface density, the temperature and the rotational velocity, a procedure to minimize errors during interpolations could be to perform them in a log-log space (which is actually an exact reconstruction in the unperturbed case). It can be done by defining $\chi=\log (r), \xi=\log (q)$ and replacing $r \rightarrow \chi$ and $q \rightarrow \xi$ in Eq. (9). However, all results presented in this work were performed using interpolations in the linear space. For comparison purposes we have run a few of them using a logarithmic interpolation method for quantities defined as a power law, showing minute differences $(\sim 0.2 \%)$ in the measured migration rates over the whole time. In more realistic cases, in which the disk cannot be modeled as a global power law, we don't expect to have a significant difference between linear or logarithmic interpolation.

Another method for un-shifting the Lindblad resonances was proposed by Baruteau \& Masset (2008b), and consists in adding to the star potential the potential of an axi-symmetric disk that has same density profile as the disk. This method is slightly more complex to implement, and in theory slightly more accurate than the one used in this work, because it matches more closely the real orbital frequencies. Nonetheless the method used here is largely sufficient to obtain migration rates within a few percent accuracy.

Regarding conservation properties, our method cannot ensure conservation of mass (and consequently neither angular momentum) since a rescale of the cells plus an interpolation procedure alters the amount of mass inside a given cell. Despite of that, the total error accumulated during a complete simulation by performing consecutive interpolations is actually small. Our tests show that the total error (compared with analytic expectations) in the worst cases, such as one with low resolution and longrange migration are up to $\sim 1 \%$ for all quantities. In the majority of cases the errors will be actually much smaller.

\section{MULTI-PLANET SIMULATIONS}

As pointed out in the introduction, part of the motivation for developing this method is to capture the large temporal and spatial ranges involved in systems with multiple planets.

In this multi-planet case, the borders of the mesh are computed similarly as was done in the single-planet case. We obtain the semi-major axis for all of them and then we calculate $r_{d}^{-}$and $r_{b}^{-}$with Eqs. (6) and (8) using the semi-major axis of the innermost planet, while the same procedure is followed for $r_{d}^{+}$and $r_{b}^{+}$, using the outermost planet.

Fig. 9 and Fig. 10 show two examples, the first corresponding to a two-planet system, while the second explores a case with three planets. Initial conditions for the bodies and the disk are detailed in the captions. In both cases the dynamical evolution is carried out for long timescales such that the final semi-major axes lie very close to the central star. The systems naturally evolve to resonant configurations, as shown by the behavior of the resonant angles.

\section{CONCLUSIONS}

We have presented a new method that allows long-term and large-scale hydrodynamical simulations of migrating planets over a grid-based Eulerian code. Our method consists in the following steps:

1. A prescription for calculating the new radial borders of the mesh (see 3.1 )

2. An interpolation method for filling the new mesh (see 3.2 ). 

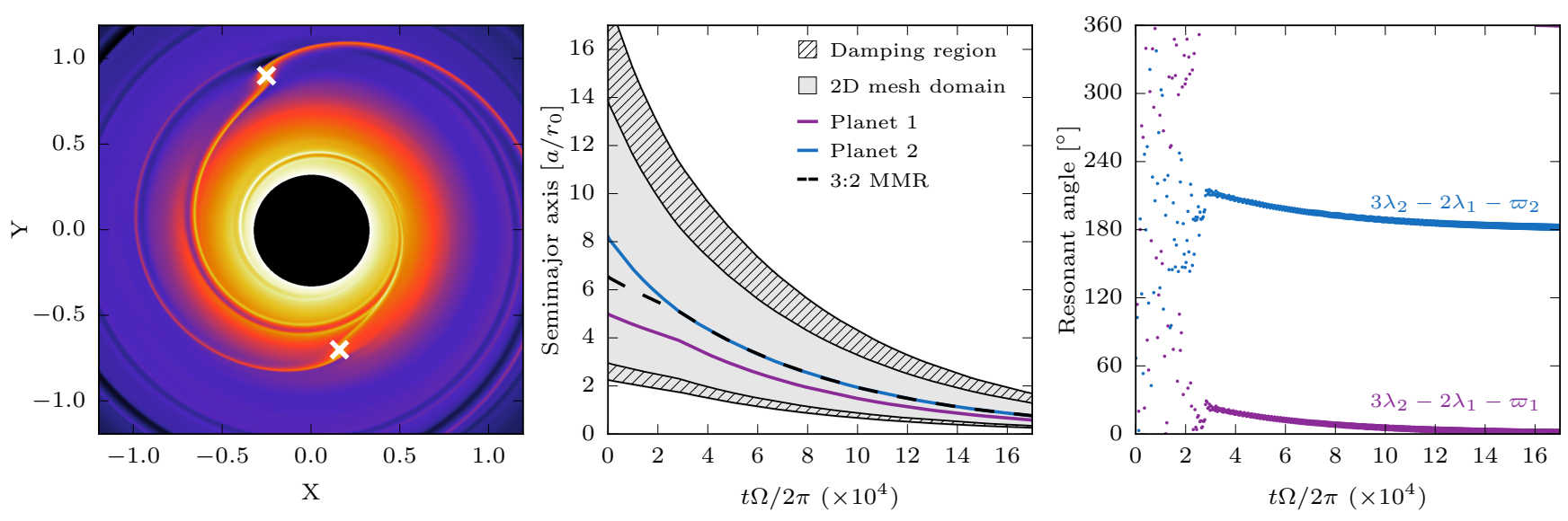

Fig. 9.- Hydrodynamical simulation for a two-planet system with masses $m_{1}=10 M_{\oplus}$ and $m_{2}=20 M_{\oplus}$. Initial semi-major axes are $a_{1}=5 \mathrm{AU}$ and $a_{2}=8.19 \mathrm{AU}$, and initial eccentricities equal to 0.05. Disk parameters are $\Sigma_{0}=400 \mathrm{gr} / \mathrm{cm}^{2}, f=0, \alpha=-0.5, h=0.05$ and $\alpha_{\nu}=4 \times 10^{-3}$. The left frame shows the disk's surface density at $t=15.53 \times 10^{4}$ orbits, where the planets are indicated with white crosses. The middle frame shows the time evolution of the semi-major axes. The light gray region corresponds to the active mesh and the hatched region to the damping zones. Approximately at $t=3 \times 10^{4}$ orbits, both planets are trapped in 3:2 MMR, after which both resonant angles begin to librate around fixed values that slowly evolve with time (right plot).
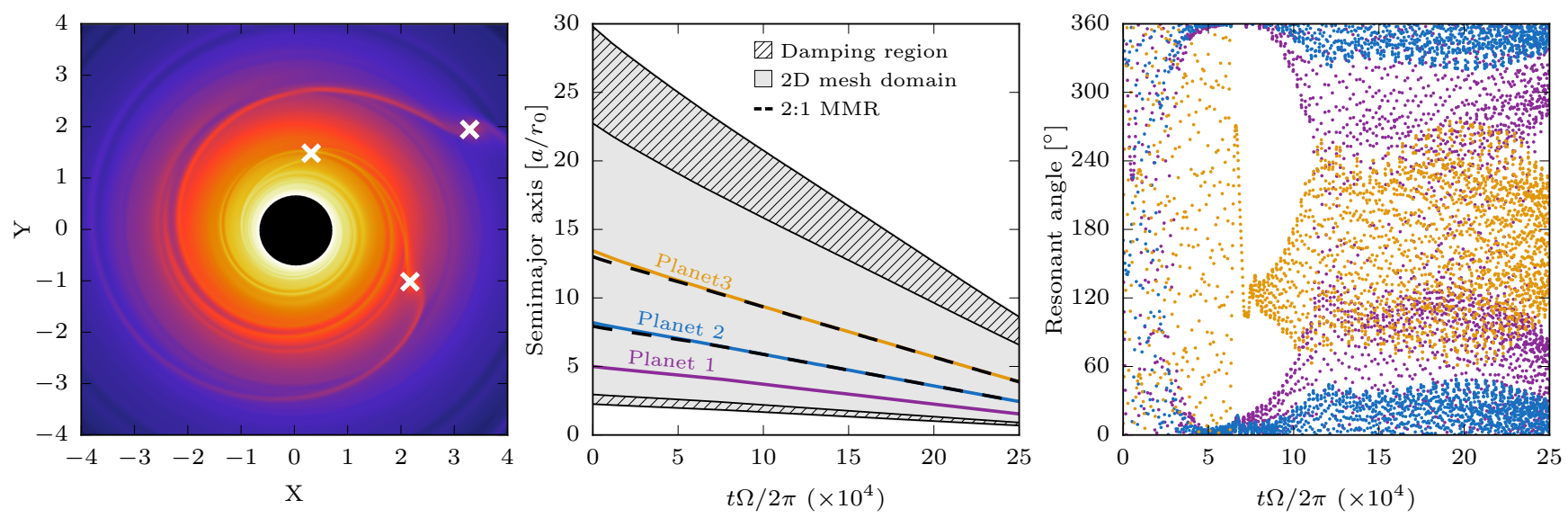

FIG. 10. - Hydrodynamical simulation of a three-planet system with masses $m_{1}=10 M_{\oplus}, m_{2}=20 M_{\oplus}$ and $m_{3}=40 M_{\oplus}$. Initial semi-major axes are $a_{1}=5 \mathrm{AU}, a_{2}=8.19 \mathrm{AU}$ and $a_{3}=13.44 \mathrm{AU}$, and initial eccentricities are equal to 0.05 . Disk parameters are $\Sigma_{0}=400 \mathrm{gr} / \mathrm{cm}^{2}, f=0.25, \alpha=-1.0, h=0.05$ and $\alpha_{\nu}=4 \times 10^{-3}$. The left frame shows the disk's surface density at $t=25 \times 10^{4}$ orbits, and the planets indicated with white crosses. The middle frame shows the time evolution of the semi-major axes. The light gray region corresponds to the active mesh and the hatched region to the damping zones. All planets are captured in successive 2:1 MMRs leading to a multiple-resonant configuration that it preserved throughout the simulation. The right plot shows the behavior of the main resonant angle of the inner pair $2 \lambda_{2}-\lambda_{1}-\varpi_{1}$ (purple), the corresponding angle of the outer pair $2 \lambda_{3}-\lambda_{2}-\varpi_{2}$ (blue), and the Laplace resonant angle $\lambda_{1}-3 \lambda_{2}+2 \lambda_{3}$ in orange.

3. Damping boundaries to avoid reflections and match the large-scale profiles (Eq. 6).

4. A method to un-shift the Lindblad resonances (see (3.4.

5. Optionally, a method to follow the global viscous evolution of the disk (see $\$ 3.3$ ).

We have validated our method with a set of tests using the public code FARGO3D and compared its results with those obtained using classical calculations with a fixed grid.

The remapping technique allows to achieve cleaner results compared to the standard case at lower computational cost. Due to the adaptive property of the algorithm described to compute the border of the mesh, the resolution naturally adapts when the planets migrate, allowing for a self-similar behavior of the system.

Our method allows for the first time to perform simulations not limited by the radial extent of the mesh. This feature enables the possibility of performing long-range and long-term hydrodynamical simulations of planetary systems, during a time comparable to the lifetime of the disk. It is important to underline that our algorithm lies on the hypothesis that we can derive the structure for the disk outside the active mesh to fill the damping zones and ghost cells.

We have also discussed how to implement the viscous evolution of the disk, which is important when simulations are long-term. However, since such simulations are also usually long-range, a realistic model for the disk should also be considered with a realistic non-power law for surface density and the temperature. 
We have discussed a possible improvement to the method, such as a logarithmic interpolation to reduce errors. However, this implementation is not strictly necessary and, in the current state, the method has a largely sufficient accuracy for practical purposes.

PBLL, XR and CB acknowledge financial support from CONICET and SECYT/UNC as well as computa- tional resources provided by IATE and CCAD (Universidad Nacional de Córdoba). FM acknowledges support from CONACyT grant 178377. We thank Ulises Amaya Olvera, Reyes García Carreón, and Jérôme Verleyen for their assistance in setting up the GPU cluster on which many of the calculations presented here have been run. We thank the referee for a very helpful report. His/her suggestions allowed us to significantly improve this work.

\section{REFERENCES}

Artymowicz, P. 1993, ApJ, 419, 155

Baruteau, C., \& Masset, F. 2008a, ApJ, 672, 1054

-. 2008b, ApJ, 678, 483

Baruteau, C., \& Masset, F. 2013, in Lecture Notes in Physics, Berlin Springer Verlag, Vol. 861, Lecture Notes in Physics, Berlin Springer Verlag, ed. J. Souchay, S. Mathis, \& T. Tokieda, 201

Baruteau, C., \& Papaloizou, J. C. B. 2013, ApJ, 778, 7 Benítez-Llambay, P., \& Masset, F. S. 2016, ApJS, 223, 11 Crida, A., Masset, F., \& Morbidelli, A. 2009, ApJ, 705, L148 Crida, A., Morbidelli, A., \& Masset, F. 2007, A\&A, 461, 1173 de Val-Borro, M., Edgar, R. G., Artymowicz, P., et al. 2006, MNRAS, 370, 529

Fabrycky, D. C., Lissauer, J. J., Ragozzine, D., et al. 2014, ApJ, 790,146
Goldreich, P., \& Tremaine, S. 1979, ApJ, 233, 857

Hansen, B. M. S., \& Murray, N. 2013, ApJ, 775, 53

Lissauer, J. J., Ragozzine, D., Fabrycky, D. C., et al. 2011, ApJS, 197,8

Martin, R. G., Lubow, S. H., Pringle, J. E., \& Wyatt, M. C. 2007, MNRAS, 378, 1589

Masset, F. 2000, A\&AS, 141, 165

Ogilvie, G. I., \& Lubow, S. H. 2002, MNRAS, 330, 950

Paardekooper, S.-J., \& Papaloizou, J. C. B. 2009, MNRAS, 394 2283

Pringle, J. E. 1981, ARA\&A, 19, 137

Rafikov, R. R. 2002, ApJ, 569, 997

Shakura, N. I., \& Sunyaev, R. A. 1973, A\&A, 24, 337

Tanaka, H., Takeuchi, T., \& Ward, W. R. 2002, ApJ, 565, 1257

Ward, W. R. 1986, Icarus, 67, 164 